\title{
Cobalt-Catalyzed Directed Alkylation of Olefinic C-H Bond with Primary and Secondary Alkyl Chlorides
}

Takeshi Yamakawa

Yuan Wah Seto

Naohiko Yoshikai ${ }^{*}$

Synlett 2015, 26, 340.

This article differs from the e-first online version in its layout. In addition, the sentence discussing Table 3 , entry 5 , was changed to read 'the desired product was not detected'. 